\title{
Radiologic Findings of Splenic Abscess: A Report of Two Cases
}

\author{
Sule M.B. ${ }^{1}$, Umar A.U. ${ }^{2}$, Gele I.H. ${ }^{3}$, Dada M.A. ${ }^{3}$, Ribah M.M. ${ }^{3}$ and Aliyu A.Z. ${ }^{3}$ \\ ${ }^{1}$ Department of Radiology, Usmanu Danfodiyo University, Sokoto. \\ ${ }^{2}$ Department of Radiology, Gombe State University, Gombe. \\ ${ }^{3}$ Department of Radiology, Usmanu Danfodiyo University Teaching Hospital, Sokoto.
}

Corresponding Author: SULE Muhammad Baba, Department of Radiology, Usmanu Danfodiyo University, Sokoto.

Received date: August 10, 2021 Accepted date: August 25, 2021 Published date: September 07, 2021

Citation: Sule MB, Umar A.U., Gele I.H., Dada M.A, Ribah MM, Aliyu AZ, (2021 Radiologic Findings of Splenic Abscess: A Report of Two Cases. J. Gastroenterology Pancreatology and Hepatobilary Disorders. 5(5) DOI: 10.31579/2641-5194/043

Copyright: (C) 2021, Sule MB, This is an open access article distributed under the Creative Commons Attribution License, which permits unrestricted use, distribution, and reproduction in any medium, provided the original work is properly cited.

\begin{abstract}
Splenic abscess is a very rare entity with an incidence of about $0.14-0.7 \%$ in autopsy studies. Splenic abscess has been reported to have a mortality rate of about $47 \%$, it has various causes but usually associated with trauma and infections of the spleen. We report two cases of splenic abscess; a child with post traumatic cause and a middle aged lady with an unknown cause.

Both cases were diagnosed of having the abscess following imaging; the first case had abdominopelvic ultrasound that showed an abscess cavity with a volume of about $11 \mathrm{mls}$ and subsequently resolved following medical treatment with intravenous antimicrobial agents. The second case however had an abdominopelvic computed tomography scan that showed a huge splenic abscess with a volume of about $1400 \mathrm{mls}$; she had a successful splenectomy. We report the radiologic findings of these cases due to the rare nature of splenic abscess as reported in the literature.
\end{abstract}

Keywords: spleen, abscess, volume, microbial

\section{Introduction}

Splenic abscess is a very rare entity with an incidence of about $0.14-0.7 \%$ in autopsy studies [1-4]. Splenic abscess though been rare, they show changing patterns of diagnosis and treatment [4]. Splenic abscess has been reported to have a mortality rate of about $47 \%$, it has various causes but usually associated with trauma and infections of the spleen [5, 6]. The incidence of splenic abscess shows an apparent increase most likely from advances in imaging studies and an increased number of immunocompromised, trauma and cancer patients [5, 7, 8]. Infective causes are the leading cause of splenic abscess, the two main contributing organisms responsible for most cases of splenic abscess are Klebsilae pneumonia and Streptococcus [4, 5]. The most common cause of splenic abscess is from hematogenous spread, and typically arise from endocarditis or seeding from some contiguous sites of infection $[9,10]$. Splenic abscess is associated with some risk factors, among which are immunocompromised status, hemoglobinopathies and diabetes mellitus $[1,11]$. The diagnosis of splenic abscess can be done early by combination of computed tomography, abdominal ultrasonography and clinical features [1, 3, 11-15]. Splenic abscess can mainly be managed by medical therapy, percutaneous aspiration (CT-guided and Abdominal ultrasonographic-guided) and splenectomy [15].

\section{Case Report}

\section{Case 1}

This is a ten-month-old infant who was referred for Abdominopelvic ultrasound following a history of fall, left hypochondrial pain, chills and rigor with easy irritability. His genotye is AA type and has no retroviral infection. The patient had tender splenomegaly at the time of presentation. The cardiovascular and respiratory systems evaluation were all within normal limits. He had much tenderness to sonographic probe pressure over the left hypochondrium.

The abdominal sonogram showed splenomegaly with a well circumscribed area of echogenicity with peripheral reduced reflectivity was demonstrated; the volume of which was $11 \mathrm{mls}$ following which a diagnosis of an abscess cavity in the spleen was made (figure 1). A plain abdominal x-ray showed sentinel loops with air fluid level in the left hypochondrium apart from the fundal gas. No history of immunosuppression was documented. The blood and abscess microscopy, culture and sensitivity yielded no growth. The blood film showed leukocytosis.

The patient got better with the use of intravenous broad spectrum antibiotics (Augmentin and intravenous metronidazole) with analgesics. He was discharged from the hospital twenty-two days later. 


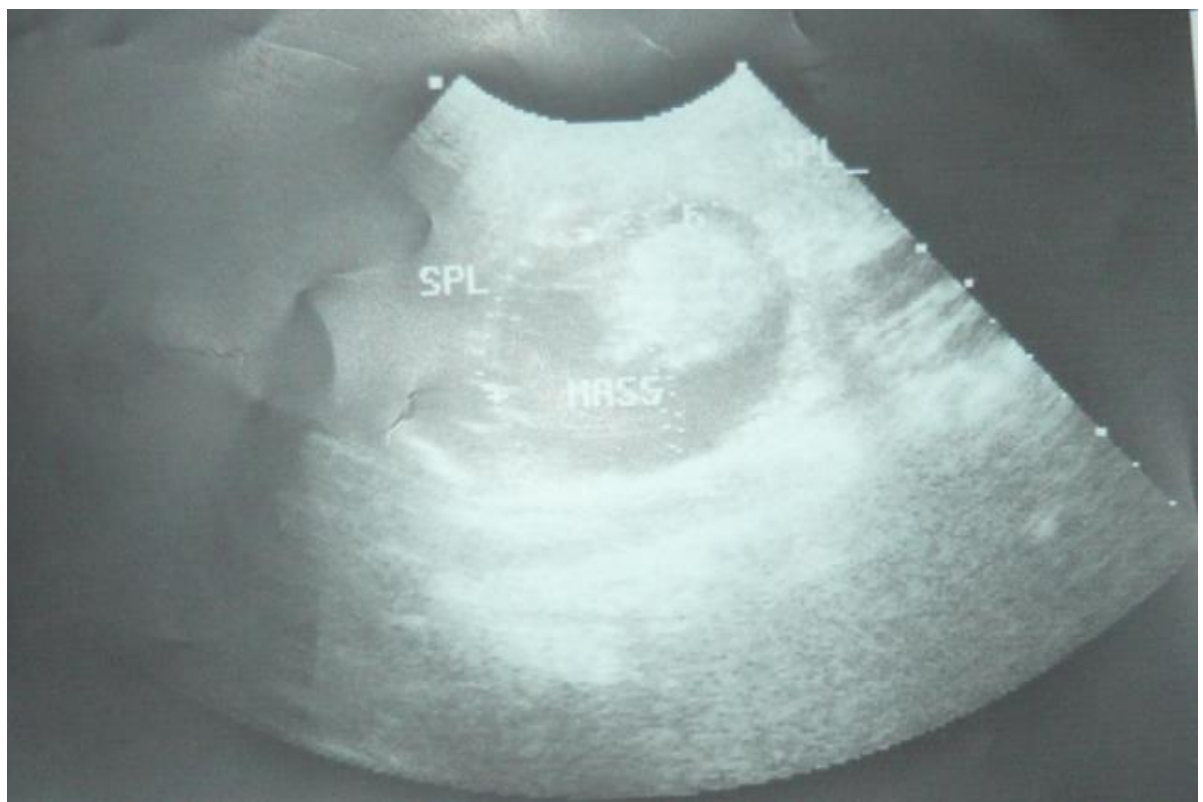

Figure 1: Ultrasonogram of the abdomen showing a well-defined area within the spleen with a central echogenic portion and a surrounding hypoechoic rim. This is an abscess cavity with a volume of about $11 \mathrm{mls}$.

\section{Case 2}

This is a 20-year-old female who was referred for Abdominopelvic computed tomography (CT) following history of recurrent left hypochondrial pain, chills and rigor with easy irritability for almost four weeks. Her genotye is AA type and has no retroviral infection. No history of fall or trauma and blood transfusion. The patient had non-tender splenomegaly at the time of presentation.

The cardiovascular and respiratory systems evaluation were all within normal limits.
The CT scan showed an extensive well circumscribed area of nonenhancing iso-density $(\mathrm{HU}=22)$ with a volume of about $1400 \mathrm{mls}$ within the substance of the spleen. It showed no debri, loculation or septum. No any other similar collection was demonstrated within the spleen; (see figures 2 a and $2 \mathrm{~b}$ ).

The blood and abscess microscopy, culture and sensitivity yielded no growth. The blood film showed leukocytosis.

The patient had initial intravenous broad spectrum antibiotics with subsequent splenectomy and got better afterwards, she was discharged home after three weeks.

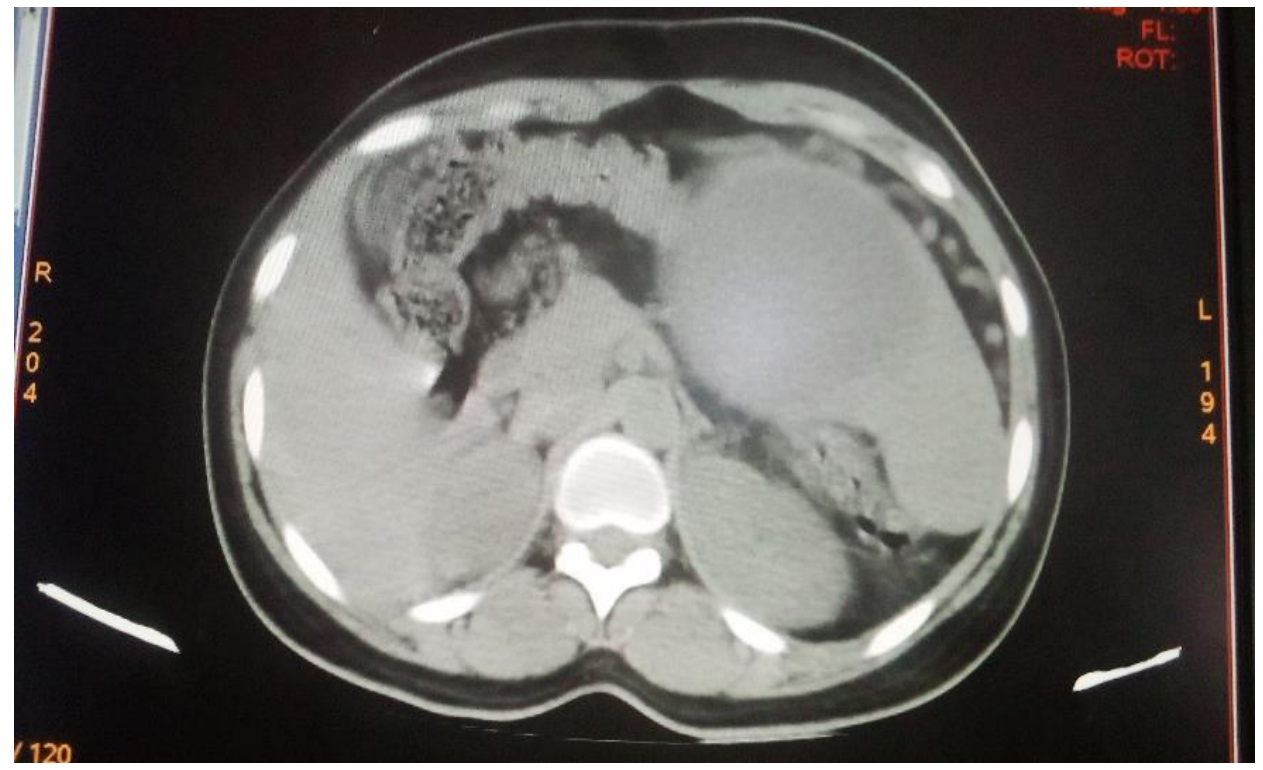

Figure 2a: early image of an abdominal CT scan showing an enlarged spleen with associated iso-dense well circumscribed area within the spleen; the splenic abscess cavity. 


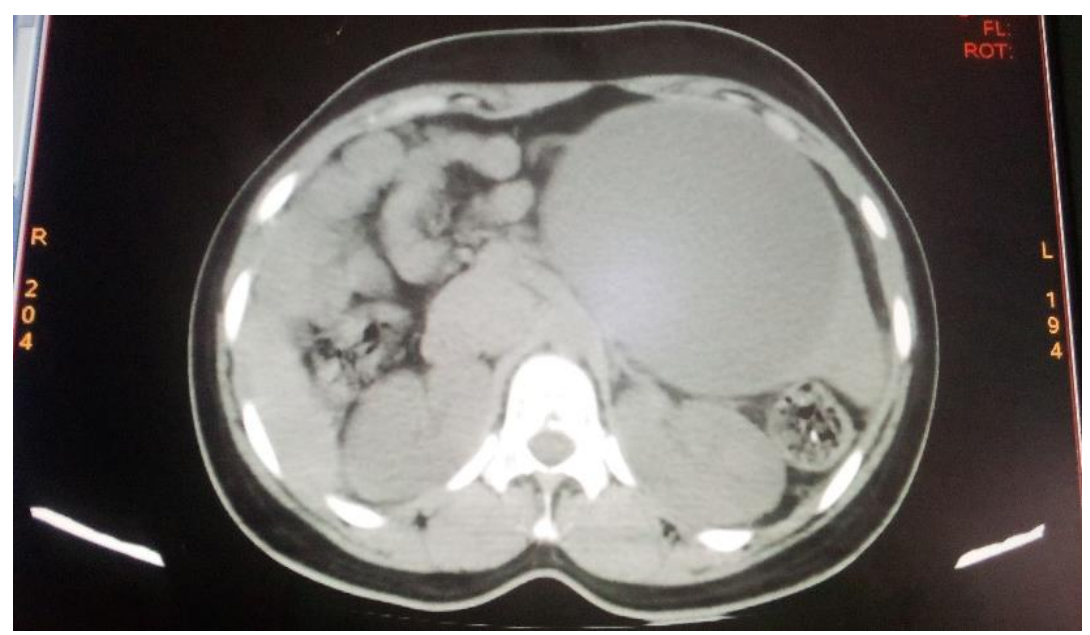

Figure 2b: Later image of an abdominal CT scan showing an enlarged spleen with associated iso-dense non-enhancing well circumscribed area within the spleen; the splenic abscess cavity with a volume of about $1400 \mathrm{mls}$.

\section{Discussion}

Splenic abscess is a rare entity and usually may follow a traumatic scenario, infective or idiopathic course. The first case had a history of trauma while the second has an idiopathic scenario. The usual complaints documented in the literature are left hypochondrial pain and discomfort, fever, chills, loss of appetite and easy fatigability to mention a few; both cases presented here had such presenting complaints respectively. Splenic abscess has equal sex distribution, the cases we had were both male and female; these cases had solitary abscess cavity though with different volumes.

Patients with splenic abscess often do have a positive growth following blood and abscess culture respectively, only in a minor cases do the culture becomes negative. Both cases presented here had no growth following culture of both blood and abscess aspirates.

Splenic abscess may be associated with other intra-abdominal abscesses especially in the liver as documented following Typhoid splenic abscesses, the two cases presented here had no any other abscess in the liver or other abdominal organs. It is documented in most literatures that splenic abscess do have a higher morbidity and mortality rates, the first case had painful and agonizing pain and longer period of debility, both cases had a favorable outcome with no mortality recorded.

Some of the literatures showed better prognosis with antimicrobial agents for single and small volume abscess cavities while splenectomy was reported to be the suitable treatment option for larger volume and multiple cavities; similar findings were documented as the first case with a smaller volume of about $11 \mathrm{mls}$ did well on antimicrobial treatment while the second case with a larger volume of about $1400 \mathrm{mls}$ had splenectomy.

\section{Conclusion}

Splenic abscess is indeed a rare entity, basic imaging and clinical features are necessary in confirming the diagnosis early to save the life of the patients. The management can either be medical or splenectomy depending on the volume and nature of the abscess with the clinical presentation of the patient.

\section{References}

1. Mei-Chun L, Chun-Ming L. (2018) Splenic Abscess: An Uncommon Entity with Potentially Life- Threatening Evolution. Can J Infect Dis Med Microbiol. 2018:8610657.
2. Westh H, Reines E, Skibsted L. (1990) splenic abscesses: a review of 20 cases. Scandinavian Journal of Infectious Diseases. 22:569573.

3. Chang KC, Chuah SK, Changchein CS. (2006) Clinical Characteristics and prognostic factors of splenic abscess: a review of 67 cases in a single medical centre of Taiwan. World Journal of Gastroenterology. 12:460-464.

4. Kidmas AT, Igun GO, Obekpa PO, Anele AA. (2003) splenic abscess in Jos. Nig J Surg Res. 5:27-31.

5. Mohammed AJ, Bandar IA, Abdallah AZ, Tagreed AA. (2016) Splenic Abscess and Management Options: Report of Four Cases. J Med Cases. 7:432-434.

6. Nelken N, Ignatius J, Skinner M, Christensen N. (1987) Changing clinical spectrum of splenic abscess. A multicenter study and review of the literature. Am J Surg. 154:27-34?

7. Teach S, Oliver GC, Canter JW. (1986) The early diagnosis of splenic abscess. Am J Surg. 52:303-307?

8. Tung CC, Chen FC, Lo CJ. (2006) splenic abscess: an easily overlooked disease? Am J Surg. 72:322-325?

9. Brook I, Frazier EH. (1998) Microbiology of liver and spleen abscesses. J Med Microbiol. 47:1075-1080.

10. Robinson SL, Saxe JM, Lucas CE, Arbulu A, Ledgerwood AM, Lucas WF. (1992) splenic abscess associated with endocarditis. Surg. 112:781-786.

11. Ng KK, Lee TY, Wan YL, et al. (2002) splenic abscess: diagnosis and management. Hepatogastroenterology. 49:567-571.

12. Nasr B, Hamida MBH, Farhat W, Othmen MB, Chhaider A, Mabrouk MB et al. (2013) Diagnosis and treatment of splenic abscess (about 11 cases). J Gastroenterol Hepatol Res 2:7867990.

13. Ferraioli G, Brunetti E, Gulizia R, Mariani G, Marone P, Filice C. (2009) Management of splenic abscess: report on 16 cases from a single centre. Int J Infect Dis. 13:524-530.

14. Krokos N, Michailidou E, Karakatsanis A, Margioulas A, Drizis Th. (1996) Splenic abscess. Hellenic J Surg. 83:148-152.

15. Lucas T, Theodora T, Georgia P, Alexandria N, Helen K, Kelekis DA. (2002) Percutaneous CT-Guided Drainage of Splenic Abscess. Am J Roent. 179:629-632. 